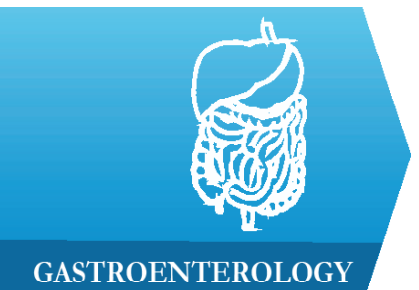

1) $2^{\text {nd }}$ Department of Internal medicine, Iuliu Hatieganu University of Medicine and Pharmacy ClujNapoca, Romania

2) Department of Nursing, Iuliu Hatieganu University of Medicine and Pharmacy Cluj-Napoca, Romania
DOI: $10.15386 / \mathrm{mpr}-2061$

Manuscript received: 25.01.2021

Received in revised form: 22.03 .2021

Accepted: 06.04.2021

Address for correspondence:

ddumitrascu@umfcluj.ro

This work is licensed under a Creative Commons Attribution-NonCommercialNoDerivatives 4.0 International License

\title{
Digestive involvement in COVID-19: what we have learned in the past 12 months
}

\author{
Vladimir Posmag ${ }^{1}$, Liliana David ${ }^{2}$, Dan L. Dumitrascu ${ }^{1}$
}

\begin{abstract}
Background and aim. The year 2020 will remain in the medical history as the year of COVID-19 pandemic. Although COVID-19 is considered mainly a respiratory disease, it is well known now that SARS-CoV-2 can affect major organs including the digestive system. This is a comprehensive review on gastrointestinal involvement in the SARS-CoV-2 infection, also the digestive damage due to COVID-19.

Methods. A literature search was undertaken in main databases of medical publications following the search items digestive or gastrointestinal or gastric or enteral and SARS-CoV-2 or COVID-19. Data on gastrointestinal symptoms and alterations in this viral condition were collected and presented. Only observational studies were included.

Results. In this review we discuss the recent information on the relationship between COVID-19 and the digestive system. The putative underlying pathogenic mechanisms of COVID-19 as well as the clinical findings in relation to gastrointestinal changes are presented.

Conclusions. SARS-CoV-2 virus can affect any part of the digestive system. Patients with chronic diseases are at greatest risk of adverse out-comes. There is a considerable risk for drug-drug interactions in patients who require a large treatment schedule.
\end{abstract}

Keywords: COVID-19, SARS-Cov-2 infection, gastrointestinal tract, liver diseases, pancreas

\section{Introduction to COVID-19}

The new coronavirus pneumonia (Coronavirus disease 2019 [COVID-19]), which is caused by severe acute respiratory syndrome coronavirus 2 (SARS-CoV-2), emerged in Wuhan, Hubei province, in early December 2019 [1] and then quickly spread throughout China and throughout the entire world, evolving into a pandemic and threatening global health. This new coronavirus, along with the severe acute respiratory syndrome coronavirus (SARS-CoV) which caused in 2002-2004 SARS epidemic and the 2012-2020 Middle East respiratory syndrome coronavirus (MERS-CoV), belong to $\beta$-coronavirus $2 \mathrm{~b}$ lineage [2]. The SARS-CoV-2 is a single stranded RNA virus, highly contagious, which spreads predominantly by respiratory droplets and aerosol, though SARS-CoV-2 has been isolated from stool, the faecaloral spread has not been confirmed. At the time of this article acceptance (March 2021), more than 120 million cases of COVID-19 were reported worldwide with over 2,6 million deaths [3].

A literature search was undertaken on main medical bibliographic databases following the search items digestive or gastrointestinal or gastric or enteral and SARS-CoV-2 or COVID-19 or Coronavirus. Data on gastrointestinal symptoms and alterations in this viral condition were collected and presented.

\section{Symptoms and clinical presentations}

Some large studies performed in UK, Wuhan, Hong-Kong, America have 
confirmed that the most common symptoms are fever, cough and shortness of breath; however, other symptom clusters were recognized including firstly, joint pain, myalgia, headache and fatigue and secondly nausea and vomiting, abdominal pain and diarrhea along with elevated liver enzymes. Almost one third of all patients reported these enteric symptoms, mostly in addition to the respiratory symptoms, and only $4 \%$ complained of enteric symptoms alone [4-6].

Diarrhea should generate awareness of a possible COVID-19 and should be investigated to reach an early diagnosis of SARS-CoV-2 infection. This factor should be considered when suspecting whether the patients are infected, instead of waiting for respiratory symptoms to appear, which might give an earlier diagnosis. Patients with SARS-CoV-2 infection, especially those with digestive symptoms, remained a longer time from onset to hospital admission and had a worse clinical outcome, compared to patients who did not suffer from these symptoms. Likewise, patients with digestive symptoms had an average time of 9 days from the onset of symptoms until admission, while patients with respiratory symptoms had an admission time of 7.3 days. This may indicate that those with digestive symptoms waited longer to be diagnosed in the hospital, as they did not suspect they were SARS-CoV-2 positive in the absence of respiratory symptoms [7].

More recently, the symptoms of dysgeusia and anosmia have been added to the core symptoms of SARS-CoV-2 with olfactory and gustatory dysfunction (Table I). Probably these symptoms appear as a result of COVID-19 neuropathy with a point of entry being by way of angiotensin-converting enzyme 2 (ACE 2) expressing cells in the olfactory epithelium and reaching the olfactory bulb via axons extending to the olfactory nucleus in the pyriform cortex [8].

Table I. Symptoms of COVID-19.

\begin{tabular}{ll} 
Common Symptoms & Digestive Symptoms \\
\hline Cough & Nausea \\
Shortness of breath & Vomiting \\
Fever & Abdominal pain \\
Joint pain & Diarrhea \\
Myalgia & Increased level of liver enzymes \\
Headache & Increased level of pancreatic enzymes
\end{tabular}

Fatigue

Dysgeusia

Anosmia

\section{Pathogenesis}

The pathogenesis of COVID-19 is now well understood, it has been shown that angiotensin-converting enzyme 2 (ACE 2) is the functional receptor of SARSCoV-2 and is critical to the cellular entry of SARS-CoV-2 [9]. This can lead to a severe host hyperimmune response in the lungs with a life-threatening cytokine storm resulting in the systemic inflammatory response syndrome [10,11]. Virus enter into epithelial cells through the spike protein on the viral coat, and this is primed by the cellular transmembrane serine protease 2 (TMPRSS-2) [11]. ACE 2 is widely distributed in human organs, including the nasal and oral mucosa, nasopharynx, lung, small intestine, colon, liver, kidney, spleen and brain. Furthermore, it is reported that ACE 2 expression is over 100-fold higher in the gastrointestinal tract (especially the colon) than in the respiratory system; according to this, digestive system, and other organs may also be affected by SARS-CoV-2 $[12,13]$.

\section{COVID-19 and the gastrointestinal tract}

Several reports based on retrospective data, from China, have demonstrated that the prevalence of gastrointetsinal (GI) symptoms in COVID-19 cases is between 11.4 and $50 \%[7,14]$. In a meta-analysis including over 4000 patients, the prevalence of GI symptoms was $17 \%$ [15]. GI symptoms presenting as an initial symptom cluster of COVID-19 infection have been reported in 3 up to $10 \%$ of adult patients and more frequently in children. The most serious GI symptom in the context of COVID-19 is severe diarrhea.

\section{Upper gut}

A recent study confirmed that ACE 2 can be found in the stratified epithelial cells of the esophagus, which may help explain the esophagitis caused by SARSCoV-2 [13]. The mucosa was observed to be damaged in the esophagus, and numerous lymphocytes and plasma cells were found to be infiltrated in the lamina propria of the stomach by histological examination [16]. Direct functional damage of the GI tract by viral interaction with the squamous and columnar epithelium mediated by ACE 2 receptor interference is likely to occur. However, the expression of ACE 2 receptors is significantly lower or absent in the esophagus and stomach as compared to intestine $[11,17,18]$. A lower expression in the esophageal and gastric as compared to intestinal mucosa is also true for the serine protease complex TMPRSS2 [20]. So, although direct viral damage is thought to occur primarily in the small and large bowel, it does not exclude an origin for abdominal symptoms referred to the upper GI tract.

\section{Lower gut}

The high expression of ACE 2 in the intestinal tract makes the small bowel and colon highly susceptible to COVID-19 infection. A recent study revealed that ACE 2 expression was more frequently found in the ileum and colon than in the lung and was especially expressed in the absorptive enterocytes of the ileum and colon, which may offer an explanation for diarrhea as a symptom in many COVID-19 patients [13].

Although COVID-19 infection can impair the digestive system in all people, the most affected persons 
might be those who are suffering from a pre-existing bowel disease, especially IBD. The onset of the SARS-CoV-2 pandemic has had a dramatic and immediate impact in the field of IBD, affecting the daily lives of patients, health care professionals, carers and the research and academic community. Clinical practice has required basic reevaluation of fundamental principles involved in patient management. It was very important to protect the safety of patients with IBD. Anxieties have been heightened by the widespread use of immune-modulator and biological agents in ulcerative colitis and Crohn's disease, when treatment goals now involve mucosal healing and full remission. It was considered that continuing immuneactive therapies, with strict attention to disinfection measures, social distancing and shielding where necessary, would be preferable to drug discontinuation, and the significant associated risk of relapse, the latter requiring investigation, hospital attendance and potentially more aggressive drug therapy or surgery [19].

\section{Covid-19 infection and liver injury}

ACE 2 probably plays a vital role in the pathogenesis of liver damage in SARS-CoV-2 infection. ACE 2 is an ACE homologue that is able to counteract the vasoconstricting effect of angiotensin (Ang) II through degrading Ang II into Ang 1-7, thus decreasing damage to the liver caused by the renin-angiotensin system [20]. ACE 2 was identified with moderate expression in healthy livers [21], but a recent single-cell RNA sequencing study of healthy liver tissues found that ACE 2 was highly expressed in cholangiocytes [22,23]. Moreover, the pathologic examination from a SARS-CoV-2 death case demonstrated mild active inflammation in the hepatic lobular portal region and moderate microvascular fatty degeneration in the liver [24], suggesting that liver damage in SARS-CoV-2 tends to be due to a secondary injury from hypoxia. It was demonstrated that the condition of patients who have coexisting liver disease might be worsened because of SARS-CoV-2 infection caused by hypoxia during cytokine storm. Choosing proper liver medications to protect liver function for SARS-CoV-2 patients is necessary.

Outcome data in chronic liver disease (CLD) patients with COVID-19 are still being collected but appear to show a greatly increased risk of death, and that the risk increases with the severity of liver disease such as increased Child-Turcotte-Pugh class or model for end stage liver disease. Also, in addition to poorer outcomes from COVID-19, frequent hepatic decompensation is reported amongst patients with pre-existing cirrhosis [25].

The impact of the SARS-CoV-2 pandemic on health care provision is large and likely to affect the care of patients with CLD with, as yet, unquantified effects on esophageal variceal surveillance, hepatocellular carcinoma surveillance, viral hepatitis care and immunosuppressive treatment regimens (e.g., greater use of budesonide in preference to prednis(ol)one) for patients with autoimmune disease [26-28].

At present, dexamethasone and remdesivir represent the two major drug treatment options for SARSCoV-2 infection [29-31]. Despite no important differences in biochemical liver injury compared with placebo in one trial [31], patients with cirrhosis and those with significant elevations of transaminase activities were excluded from recruitment to the major study on remdesivir, and patients with decompensated cirrhosis and transaminase elevations $>5 \times$ ULN are recommended not to receive remdesivir $[29,30]$. Reactivation of chronic hepatitis B with dexamethasone therapy must be considered, especially in the areas where the virus is endemic [31,32]. The reported benefit from corticosteroid therapy highlights the uncertainty regarding the relative risk of COVID-19 to CLD patients which require immunosuppression, particularly those with autoimmune hepatitis, where data to guide care are urgently needed [29].

Drug toxicity has been the first mechanism for SARS-CoV-2 associated liver injury, which also indicates that liver damage may be secondary. Some drugs used in SARS-CoV-2 patients can cause liver function damage, including antipyretics, traditional Chinese medicine, and antiviral drugs. In a SARS associated report, 7 of 41 patients with severe acute respiratory syndrome treated with Kaletra experienced liver dysfunction, and 1 patient had severe liver dysfunction and thus had to discontinue antiviral treatment [33]. In a Singapore study, 3 of 5 SARS-CoV-2 infected patients with ritonavir-lopinavir treatment developed liver function disturbance [34]. Nevertheless, little is known concerning the incidence of hepatotoxicity of multiple drugs used in COVID-19 and much more efforts should be made toward future studies regarding this concern.

\section{Covid-19 infection and pancreatic disease}

The prevalence of COVID-19 presenting as acute pancreatitis is $0.27 \%$ among patients hospitalized with SARS-CoV-2 infection. Idiopathic pancreatitis was the most common etiology in this group of patients $(69 \%)$ compared to $21 \%$ in patients who were SARS-CoV-2 negative. Black and Hispanic races with pancreatitis were more likely to be diagnosed with SARS-CoV-2 infection after multivariate analysis [35].

A mechanism responsible for the induction of mild pancreatic injury, a direct cytotoxic viral involvement is possible as soon as the expression of ACE 2 receptors has been described in the pancreas, and particularly in pancreatic islets [18].

In a small clinical series which included patients with COVID-19 pneumonia, pancreatic damage with mild clinical manifestation was detected in 9 of 52 patients $(17 \%)$. In these patients, the authors observed a higher 
incidence of diarrhea and loss of appetite with increased pancreatic serum enzyme elevation, which they explained as pancreatic injury [36]. Pancreatic serum enzyme elevations must be interpreted with caution. In the absence of severe abdominal pain, such findings can't be attributed to pancreatic injury and pancreatitis, without appropriate investigations. Otherwise, it is not possible to exclude minor, subclinical vascular or cellular damage which isn't detectable by radiological imaging. Further explanations for elevated pancreatic enzyme measurements in serum might result from an imbalance between production and degradation of lipase and amylase or an increased absorption of these macromolecular enzymes by a leaky gut [37]. It may be more probable that secondary pancreatic enzyme abnormalities can be interpreted as a non-specific consequance to the severe illness and drugs prescribed to treat.

Concerning pancreatitis scores, these were significantly higher in the COVID-19 positive patients. Alcoholic and idiopathic acute pancreatitis were predominant in the non-infected patients and significantly different from the COVID-19 cohort, and mortality was significantly higher in those with existing COVID-19 who displayed more multi-organ and persistent organ failure [38].

\section{Pharmacotherapeutic considerations for GI treatments in COVID-19 patients}

In this section we would like to highlight the impact of SARS-CoV-2 infection and its treatments on the management of GI and liver diseases. According to two systematic reviews $[39,40]$, the pooled prevalence of diarrhea in patients with SARS-CoV-2 infection is 7.4$7.7 \%$, and $18.3 \%$ in studies from outside China. Diarrhea usually appeared during hospitalization, with a minority presenting on admission. If stool cultures for Clostridium difficile were negative in all patients and calprotectin or fecal leucocytes values not presented, diarrhea may have been related to drug treatment(s) [40]. Some drugs against SARS-CoV-2 such as antivirals, especially ritonavirlopinavir combinations, antimicrobials and antimalarials can induce nausea, diarrhea and also vomiting. Since drug discontinuation is not always possible, antidiarrheal compounds should be given to prevent dehydration and electrolyte disturbances. However, to avoid delaying viral clearance [39,41], antisecretory compounds (e.g., racecadotril) should be preferred to combination (antisecretory and antimotility) agents like loperamide. Similarly, nausea and vomiting (pooled prevalence 4.6$7.8 \%$ ) $[5,39]$ need treatment to prevent dehydration and avoid interference with non-invasive ventilation, when needed. Anti-emetics should be used with caution since these drugs prolong the QTc interval [41], especially when combined with other drugs being used for SARS-
CoV-2 infection such as hydroxychloroquine, chloroquine and azithromycin. Corticosteroids and mesalazine are risk factors for severe SARS-CoV-2 infection among IBD patients, but this is not the case for TNF antagonists [42], such as infliximab which seems useful to treat both the underlying inflammation and COVID-19 pneumonia by countering the cytokine storm $[43,44]$. Based on two systematic reviews with meta-analysis [40,45], liver function tests (bilirubin, aspartate and alanine aminotransferases) were abnormal in $15-19 \%$ of patients with SARS-CoV-2 infection. Available evidence suggests that, in this group of patients, liver injury can result from direct pathogenic effects by the virus, systemic inflammation with a complicated disease course or toxicity from commonly used medications [46].

During the ongoing pandemic of SARS-CoV-2, more attention was paid to the balance of risks and benefits associated with proton pump inhibitors. Studies concerning this problem were developed worldwide to find out the relation between PPIs use and COVID-19. Most of them have shown that patients taking PPIs are at increased risk for severe clinical outcomes of COVID-19 but not susceptible to SARS-CoV-2 infection. This suggests that physicians need to make benefit-risk assessments in the management of acid-related diseases amid the COVID-19 pandemic [47].

The risk for drug-drug interactions is considerable in patients seriously ill with SARS-CoV-2 infection who often require mechanical ventilation and life support. Some repurposed drugs used against SARS-CoV-2 can cause or aggravate some of the COVID-19 related gastrointestinal symptoms and may also induce liver injury [48].

\section{Endoscopic interventions in COVID-19 patients}

The large number of SARS-CoV-2 patients has led to radical changes in all endoscopy services, as the clinicians have tried to carry on offering patients what are often life-saving procedures. Most of gastrointestinal procedures have a high risk of aerosol contamination of COVID-19 to all medical staff involved in. Therefore it was necessary to reduce as much as possible the number of performed procedures in order to maintain the safety of patients and staff. The virus is not enough blocked by the use of standard surgical masks and all guidelines recommend the use of level 2 personal protective equipment which include FFP3 masks, head coverings, visors, long-sleeved fluid-resistant gowns, two pairs of gloves and shoe coverings [49]. The reduced endoscopy units continue to expose patients to the risk of harm and worse outcomes due to long waits for procedures. So, the importance of finding a reasonable solution is significant. Thus, maintaining a balance between protecting healthcare workers and patients from taking 
the SARS-CoV-2 infection on one hand and providing timely and effective clinical care on the other will become increasingly important as the pandemic persists in order to save lives [50].

\section{Discussion}

In order to provide more important information about the digestive involvement in SARS-CoV-2 infection we have analyzed recent published articles based on metaanalysis and discussed the results which have concluded that the most significant GI symptoms were anorexia and diarrhea, the most significant abnormal liver function was increased ALT and severe patients were more likely to have GI symptoms and abnormal liver function [51]. Also, it has been confirmed one more time that digestive symptoms and liver injury are not uncommon in patients with COVID-19 and the presence of underlying chronic liver disease is significantly associated with more severe COVID-19 infections and mortality. Thus, increased attention should be paid to the care of this unique group of patients $[51,52]$.

\section{Conclusions}

The SARS-CoV-2 virus can affect the digestive system as well as other organs in human body. The most common digestive symptoms in Covid-19 infection are diarrhea, nausea, vomiting, abdominal pain, along with elevated liver enzymes, which in rare cases appear as initial symptoms.

Digestive symptoms must be treated with caution in the early stage of COVID-19, also dynamic monitoring of liver function and cytokines is imperative during clinical practice to reduce the complications and mortality of SARS-CoV-2 infection.

The use of some medications for managing COVID-19 may be associated with GI symptoms, and others may lead to serious adverse events or drug-drug intercations. The risks of immune-modulatory therapies in patients with IBD or CLD continue to be carefully studied, but withdrawal of treatment in patients in remission is not necessary in view of the real and significant risks of relapse.

The relationship between the digestive system and COVID-19 should be further explored in future related studies.

\section{References}

1. Huang C, Wang Y, Li X, Ren L, Zhao J, Hu Y, et al. Clinical features of patients infected with 2019 novel coronavirus in Wuhan, China. Lancet 2020;395:497-506.

2. Wang D, Hu B, Hu C, Zhu F, Liu X, Zhang J, et al. Clinical characteristics of 138 hospitalized patients with 2019 novel coronavirus-infected pneumonia in Wuhan, China. JAMA. 2020;323:1061-1069.
3. WHO Coronavirus Disease (COVID-19) Dashboard. Available from: https://covid19.who.int/html

4. Docherty AB, Harrison EM, Green CA, Hardwick HE, Pius R, Norman L, et al. Features of 20133 UK patients in hospital with covid-19 using the ISARIC WHO Clinical Characterisation Protocol: prospective observational cohort study. BMJ. 2020;369:m1985.

5. Han C, Duan C, Zhang S, Spiegel B, Shi H, Wang W, et al. Digestive symptoms in COVID-19 patients with mild disease severity: clinical presentation, stool viral RNA testing, and outcomes. Am J Gastroenterol. 2020;115:916-923.

6. Tong JY, Wong A, Zhu D, Fastenberg JH, Tham T. The Prevalence of Olfactory and Gustatory Dysfunction in COVID-19 Patients: A Systematic Review and Metaanalysis. Otolaryngol Head Neck Surg. 2020;163:3-11.

7. Pan L, Mu M, Yang P, Sun Y, Wang R, Yan J, et al. Clinical characteristics of COVID-19 patients with digestive symptoms in Hubei, China: a descriptive, cross-sectional, multicenter study. Am J Gastroenterol. 2020;115:766-773.

8. Wu Y, Xu, Chen Z, Duan J, Hashimoto K, Yang, L, et al. Nervous system involvement after infection with Covid-19 and other coronaviruses. Brain Behav Immun. 2020;87:18-22.

9. Li W, Moore MJ, Vasilieva N, Sui J, Wong SK, Berne MA, et al. Angiotensin-converting enzyme 2 is a functional receptor for the SARS coronavirus. Nature. 2003;426:450-454.

10. Siddiqi HK, Mehra MR. COVID-19 illness in native and immunosuppressed states: A clinical-therapeutic staging proposal. J Heart Lung Transplant. 2020;39:405-407.

11. Hoffmann M, Kleine-Weber H, Schroeder S, Krüger N, Herrler T, Erichsen S, et al. SARS-CoV-2 Cell Entry Depends on ACE2 and TMPRSS2 and Is Blocked by a Clinically Proven Protease Inhibitor. Cell. 2020;181:271-280.e8.

12. ACE2 angiotensin converting enzyme 2 [Homo sapiens (human)]. In Gene/PubMed Available from: https://www. ncbi.nlm.nih.gov/gene? $\mathrm{Db}=$ gene $\& \mathrm{Cmd}=$ DetailsSearch $\& \mathrm{Te}$ $\mathrm{rm}=59272$

13. Zhang H, Kang Z, Gong H, Xu D, Wang J, Li Z, et al. The digestive system is a potential route of 2019-nCov infection: a bioinformatics analysis based on single-cell transcriptomes. bioRxiv. Published online January 31, 2020. Available from: https://www.biorxiv.org/content/10.1101/2020.01.30.92780 6v1.full.pdf

14. Jin X, Lian JS, Hu JH, Gao J, Zheng L, Zhang YM, et al. Epidemiological, clinical and virological characteristics of 74 cases of coronavirus-infected disease 2019 (COVID-19) with gastrointestinal symptoms. Gut. 2020;69:1002-1009.

15. Cheung KS, Hung IFN, Chan PPY, Lung KC, Tso E, Liu R, et al Gastrointestinal manifestations of SARS-CoV-2 infection and virus load in fecal samples from a Hong Kong cohort: systematic review and meta-analysis. Gastroenterology. 2020;159:81-95.

16. Xiao F, Tang M, Zheng X, Liu Y, Li X, Shan H. Evidence for Gastrointestinal Infection of SARS-CoV-2. Gastroenterology. 2020;158:1831-1833.e3

17. Zhang H, Kang Z, Gong H, Xu D, Wang J, Li Z, et al. Digestive system is a potential route of COVID-19: an analysis of single-cell coexpression pattern of key proteins 
in viral entry process. Gut. 2020;69:1010-1018.

18. Muus C, Luecken MD, Eraslan G, Waghray A, Heimberg $\mathrm{G}$, Sikkema L, et al. Integrated analyses of single-cell atlases reveal age, gender, and smoking status associations with cell type-specific expression of mediators of SARSCoV-2 viral entry and highlights inflammatory programs in putative target cells. bioRxiv 2020: 2020.04.19.049254. Available from: http:// biorxiv.org/content/ early/2020/04/21/2020.04.19.049254

19. Kennedy NA, Jones GR, Lamb CA, Appleby R, Arnott I, Beattie RM, et al. British Society of Gastroenterology guidance for management of inflammatory bowel disease during the COVID-19 pandemic. Gut. 2020;69:984-990.

20. Paizis G, Tikellis C, Cooper ME, Schembri JM, Lew RA, Smith AI, et al. Chronic liver injury in rats and humans upregulates the novel enzyme angiotensin converting enzyme 2. Gut. 2005;54:1790-1796.

21. Donoghue M, Hsieh F, Baronas E, Godbout K, Gosselin M, Stagliano N, et al. A novel angiotensin-converting enzymerelated carboxypeptidase (ACE2) converts angiotensin I to angiotensin 1-9. Circ Res. 2000;87:E1-E9.

22. Chai X, Hu L, Zhang Y, Han W, Lu Z, Ke A et al. Specific ACE2 expression in cholangiocytes may cause liver damage after 2019-nCoV infection. bioRxiv; 2020. Available from: https://www.biorxiv.org/content/10.1101/2020.02.03.93176 6v1.full.pdf

23. Yao XH, Li TY, He ZC, Ping YF, Liu HW, Yu SC, et al. A pathological report of three COVID-19 cases by minimally invasive autopsies. Zhonghua Bing Li Xue Za Zhi 2020;49:411-417.

24. Xu Z, Shi L, Wang Y, Zhang J, Huang L, Zhang C, et al. Pathological findings of COVID-19 associated with acute respiratory distress syndrome. Lancet Respir Med. 2020;8: $420-422$.

25. Iavarone M, D'Ambrosio R, Soria A, Triolo M, Pugliese N, Del Poggio $\mathrm{P}$, et al. High rates of 30-day mortality in patients with cirrhosis and COVID-19. J Hepatol. 2020;73:10631071.

26. Blach S, Kondili LA, Aghemo A, Cai Z, Dugan E, Estes C, et al. Impact of COVID-19 on global HCV elimination efforts. J Hepatol. 2020;74:31-36.

27. Lleo A, Invernizzi P, Lohse AW, Aghemo A, Carbone M. Management of patients with autoimmune liver disease during COVID-19 pandemic. J Hepatol. 2020; 73:453-455.

28. Tapper EB, Asrani SK. The COVID-19 pandemic will have a long-lasting impact on the quality of cirrhosis care. J Hepatol. 2020;73:441-445.

29. Grein J, Ohmagari N, Shin D, Diaz G, Asperges E, Castagna A, et al. Compassionate Use of Remdesivir for Patients with Severe Covid-19. N Engl J Med. 2020;382:2327-2336.

30. Wang $\mathrm{Y}$, Zhang $\mathrm{D}, \mathrm{Du} \mathrm{G}, \mathrm{Du} \mathrm{R}$, Zhao J, Jin $\mathrm{Y}$, et al. Remdesivir in adults with severe COVID- 19: a randomised, double-blind, placebo-controlled, multicentre trial. Lancet. 2020;395:1569-1578.

31. RECOVERY Collaborative Group, Horby P, Lim WS, Emberson JR, Mafham M, Bell JL, et al. Dexamethasone in Hospitalized Patients with Covid-19. N Engl J Med.
2021;384:693-704.

32. Reddy KR, Beavers KL, Hammond SP, Lim JK, FalckYtter YT; American Gastroenterological Association Institute. American Gastroenterological Association Institute guideline on the prevention and treatment of hepatitis B virus reactivation during immunosuppressive drug therapy. Gastroenterology. 2015;148:215-219; quiz e16-e17.

33. Chu CM, Cheng VC, Hung IF, Wong MM, Chan KH, Chan $\mathrm{KS}$, et al. Role of lopinavir/ritonavir in the treatment of SARS: initial virological and clinical findings. Thorax. 2004;59:252-256.

34. Young BE, Ong SWX, Kalimuddin S, Low JG, Tan SY, Loh J, et al. Epidemiologic Features and Clinical Course of Patients Infected with SARS-CoV-2 in Singapore. JAMA. 2020;323:1488-1494.

35. Inamdar S, Benias PC, Liu Y, Sejpal DV, Satapathy SK, Trindade AJ, et al. Prevalence, Risk Factors, and Outcomes of Hospitalized Patients with Coronavirus Disease 2019 Presenting as Acute Pancreatitis. Gastroenterology. 2020;159:2226-2228.e2.

36. Wang F, Wang H, Fan J, Zhang Y, Wang H, Zhao Q. Pancreatic Injury Patterns in Patients with Coronavirus Disease 19 Pneumonia. Gastroenterology. 2020;159:367-370.

37. Tositti G, Fabris P, Barnes E, Furlan F, Franzetti M, Stecca C, et al. Pancreatic hyperamylasemia during acute gastroenteritis: incidence and clinical relevance. BMC Infect Dis. 2001;1:18.

38. Aloysius MM, Thatti A, Gupta A, Sharma N, Bansal P, Goyal H. COVID-19 presenting as acute pancreatitis. Pancreatology. 2020;20:1026-1027.

39. Parasa S, Desai M, Thoguluva Chandrasekar V, Patel HK, Kennedy KF, Roesch T, et al. Prevalence of Gastrointestinal Symptoms and Fecal Viral Shedding in Patients With Coronavirus Disease 2019: A Systematic Review and Metaanalysis. JAMA Netw Open. 2020;3:e2011335.

40. Sultan S, Altayar O, Siddique SM, Davitkov P, Feuerstein JD, Lim JK, et al. AGA Institute Rapid Review of the Gastrointestinal and Liver Manifestations of COVID-19, Meta-Analysis of International Data, and Recommendations for the Consultative Management of Patients with COVID-19. Gastroenterology. 2020;159:320-334.e27.

41. Frommeyer G, Fischer C, Ellermann C, Lange PS, Dechering DG, Kochhäuser S, et al. Severe Proarrhythmic Potential of the Antiemetic Agents Ondansetron and Domperidone. Cardiovasc Toxicol. 2017;17:451-457.

42. Brenner EJ, Ungaro RC, Gearry RB, Kaplan GG, KissousHunt M, Lewis JD, et al. Corticosteroids, but not TNF antagonists, are associated with adverse COVID-19 outcomes in patients with inflammatory bowel diseases: results from an international registry. Gastroenterology. 2020;159:481-491.e3.

43. Bezzio C, Saibeni S, Variola A, Allocca M, Massari A, Gerardi V, et al. Outcomes of COVID-19 in 79 patients with IBD in Italy: an IG-IBD study. Gut. 2020;69:1213-1217.

44. Dolinger MT, Person H, Smith R, Jarchin L, Pittman $\mathrm{N}$, Dubinsky $\mathrm{MC}$, et al. Pediatric Crohn Disease and Multisystem Inflammatory Syndrome in Children (MIS-C) 
and COVID-19 Treated with Infliximab. J Pediatr Gastroenterol Nutr. 2020;71:153-155.

45. Mao R, Qiu Y, He JS, Tan JY, Li XH, Liang J, et al. Manifestations and prognosis of gastrointestinal and liver involvement in patients with COVID-19: a systematic review and meta-analysis [published correction appears in Lancet Gastroenterol Hepatol. 2020;5(7):e6]. Lancet Gastroenterol Hepatol. 2020;5:667-678.

46. Garrido I, Liberal R, Macedo G. Review article: COVID-19 and liver disease-what we know on 1st May 2020. Aliment Pharmacol Ther. 2020;52:267-75.

47. Marks DJB. Time to halt the overprescribing of proton pump inhibitors. Clinical Pharmacist. 2016:8. doi:10.1211/ CP.2016.20201548.

48. Hunt RH, East JE, Lanas A, Malfertheiner P, Satsangi J, Scarpignato C, et al. COVID-19 and Gastrointestinal Disease: Implications for the Gastroenterologist. Dig Dis. 2021;39:119-139.
49. Chiu PWY, Ng SC, Inoue H, Reddy DN, Ling Hu E, Cho JY, et al. Practice of endoscopy during COVID-19 pandemic: position statements of the Asian Pacific Society for Digestive Endoscopy (APSDE-COVID statements). Gut. 2020;69:991-996.

50. Lui RN, Wong SH, Sánchez-Luna SA, Pellino G, Bollipo S, Wong MY, et al. Overview of guidance for endoscopy during the coronavirus disease 2019 pandemic. J Gastroenterol Hepatol. 2020;35:749-759.

51. Dong ZY, Xiang BJ, Jiang M, Sun MJ, Dai C. The Prevalence of Gastrointestinal Symptoms, Abnormal Liver Function, Digestive System Disease and Liver Disease in COVID-19 Infection: A Systematic Review and Meta-Analysis. J Clin Gastroenterol. 2021;55:67-76.

52. Kumar-M P, Mishra S, Jha DK, Shukla J, Choudhury A, Mohindra R, et al. Coronavirus disease (COVID-19) and the liver: a comprehensive systematic review and meta-analysis. Hepatol Int. 2020;14:711-722. 